University of Denver

Digital Commons @ DU

Electrical and Computer Engineering: Graduate

Student Scholarship

Department of Electrical and Computer

Engineering

2018

\title{
A Pilot Study on Facial Expression Recognition Ability of Autistic Children Using Ryan, a Rear-Projected Humanoid Robot
}

\author{
Farzaneh Askari \\ University of Denver, farzaneh.askari@du.edu \\ Huanghao Feng \\ University of Denver, howard.k.feng@gmail.com \\ Timothy D. Sweeny \\ University of Denver, Timothy.Sweeny@du.edu \\ Mohammad H. Mahoor \\ University of Denver, mohammad.mahoor@du.edu
}

Follow this and additional works at: https://digitalcommons.du.edu/ritchie_ece_gstudent_scholarship

Part of the Cognition and Perception Commons, Electrical and Computer Engineering Commons, and the Robotics Commons

\section{Recommended Citation}

Askari, Farzaneh; Feng, Huanghao; Sweeny, Timothy D.; and Mahoor, Mohammad H., "A Pilot Study on Facial Expression Recognition Ability of Autistic Children Using Ryan, a Rear-Projected Humanoid Robot" (2018). Electrical and Computer Engineering: Graduate Student Scholarship. 3.

https://digitalcommons.du.edu/ritchie_ece_gstudent_scholarship/3

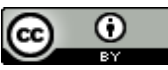

This work is licensed under a Creative Commons Attribution 4.0 License.

This Conference Proceeding is brought to you for free and open access by the Department of Electrical and Computer Engineering at Digital Commons @ DU. It has been accepted for inclusion in Electrical and Computer Engineering: Graduate Student Scholarship by an authorized administrator of Digital Commons @ DU. For more information, please contact jennifer.cox@du.edu,dig-commons@du.edu. 


\section{A Pilot Study on Facial Expression Recognition Ability of Autistic Children Using Ryan, a Rear-Projected Humanoid Robot}

\section{Comments}

Conference paper presented at RO-MAN 2018 - IEEE International Conference on Robot and Human Interactive Communication, Aug. 27-31, 2018 - Nanjing, China.

Published paper may be found at: 


\title{
A Pilot Study on Facial Expression Recognition Ability of Autistic Children Using Ryan, A Rear-Projected Humanoid Robot
}

\author{
Farzaneh Askari $^{1}$, Haunghao Feng ${ }^{1}$, Timothy D. Sweeny ${ }^{2}$, Mohammad H. Mahoor ${ }^{1,3}$
}

\begin{abstract}
Rear-projected robots use computer graphics technology to create facial animations and project them on a mask to show the robot's facial cues and expressions. These types of robots are becoming commercially available, though more research is required to understand how they can be effectively used as a socially assistive robotic agent. This paper presents the results of a pilot study on comparing the facial expression recognition abilities of children with Autism Spectrum Disorder (ASD) with typically developing (TD) children using a rearprojected humanoid robot called Ryan. Six children with ASD and six TD children participated in this research, where Ryan showed them six basic expressions (i.e. anger, disgust, fear, happiness, sadness, and surprise) with different intensity levels. Participants were asked to identify the expressions portrayed by Ryan. The results of our study show that there is not any general impairment in expression recognition ability of the ASD group comparing to the TD control group; however, both groups showed deficiencies in identifying disgust and fear. Increasing the intensity of Ryan's facial expressions significantly improved the expression recognition accuracy. Both groups were successful to recognize the expressions demonstrated by Ryan with high average accuracy.
\end{abstract}

\section{INTRODUCTION}

Children with Autism Spectrum Disorder (ASD) experience deficiency in verbal and non-verbal social skills. For most, ASD is a lifelong disorder, with long lasting symptoms from early childhood through adulthood [1]. Although, there is no known cure for ASD, research has demonstrated that those individuals who received behavior intervention during early ages exhibit improvements in communication and social skills in adulthood [2]. Therefore, it is of crucial importance to study and treat ASD in early ages.

The Diagnostic and Statistical Manual of Mental Disorders ( $^{\text {th }}$ edition; DSM-5; American Psychiatric Association, 2013) described ASD symptoms as deficits in social interaction, communication, and the presence of restricted, repetitive patterns of behavior, interests, or activities [3]. Although facial expression recognition and emotion perception are not main parts of the ASD definition, they are regarded as common shortfalls of individual with ASD [4], that prevent individual with ASD from perceiving other's mental state and regulating their behaviors accordingly. In other words, emotion perception and expression recognition deficiencies can considerably limit social development in individuals with ASD.

\footnotetext{
${ }^{1}$ Farzaneh Askari, Haunghao Feng, and Dr. Mohammad H. Mahoor: Department of Electrical and Computer Engineering, University of Denver, Denver, CO 80208, farzaneh.askari@du.edu, howard.k.finn@gmail.com, mmahoor@du.edu ${ }^{2}$ Dr. Timothy D. Sweeny: Department of Psychology,
}

Research has demonstrated that many children with ASD exhibit comfort and interest toward technology and robots $[5,6]$; as a result, the field of Socially Assistive Robots (SAR) has been widely studied [7]. Since children with ASD exhibit less anxiety and more comfort in more predictable (systematic) environments [8], robots can be used effectively to teach social skills to them because of their simplicity and predictability. There have been several socially assistive robots developed with emotionally expressive faces. Some of them such as KASPAR [9] and Tito [10] have more simplified faces to reduce sensory overload and anxiety [5]. In contrast, some other humanoid robots such as FACE [11] and Zeno [12] can demonstrate nearly realistic human facial expressions. Humanoid facially expressive robots are effective tools to target facial expression recognition and emotion perception in children with ASD. They are capable of expressing human like expressions and keeping children comfortable and engaged in a social environment. The idea that children with ASD suffer from emotion recognition deficiency is presumed [13,14]; however, there are studies $[15,16]$ that cast doubt on the idea of a general emotionrecognition deficiency in children with ASD; instead, they suggest ASD children may perform worse, comparing to their Typically Developing (TD) peers, in recognizing some expressions out of six basic expressions outlined by P. Ekman [17]. Moreover, as [18] noted, children with ASD are not always impaired in recognizing expression with $100 \%$ intensity. Therefore, it is important to first study the deficiency, if one exists, in recognizing different expression intensities; and second, to use effective tools (e.g. SAR) to target and treat it.

There have been many studies in using SAR as a tool to teach social skills and emotion recognition to children with ASD. Keepon is a non-humanoid robot with snowman-like body made of silicon rub, which is able to express excitement, pleasure and fear emotions with body movement [19]. A study with a three-year-old autistic girl and a group of twentyfive TD children in the age range of 1-3 showed the success of Keepon to improve some of the social skills such as eye contact, joint attention, emotional expression, and turn-taking in both groups after several intervention sessions with Keepon[20].

An example of using humanoid robots to teach social skills to autistic children, is a study using KASPAR [9], which is a child-size male robot with active arms, hands, and head.

University of Denver, Denver, CO 80208 Timothy.Sweeny@du.edu ${ }^{3}$ Dr. Mohammad H. Mahoor: DreamFace Technologies, LLC http://dreamfacetech.com/ 
KASPAR can open and close its mouth and eyes. In another study [21] KASPAR is used as a therapeutic tool for a 16year-old boy, who was diagnosed with severe autism and could not tolerate other children. The intervention sessions improved his skills such as imitation, eye contact, joint attention, and turn-taking. Besides, the child showed interest toward the robot's eyes, eye lids and face. This interest led to the child later touching his own face and eyes as well as those of his therapist.

Another study used FACE [11], a female android robot. The robot's face is made of skin-like silicon rubber, which enables the robot to show six basic expressions (i.e. anger, disgust, fear, happiness, sadness, and surprise). FACE has been used in [22] to target emotional behavior. The study included four subjects with high functioning autistic individuals in the range of seven to twenty-years-old. All subjects demonstrated improvement in emotional behavior at the end of the intervention sessions. Additionally, participants showed a spontaneous ability of imitating the head movements and facial expressions of the robot.

Recent studies [23,24] used Zeno R50 [12], which is a child-size male robot with active arms and legs, and the ability to express six basic facial expressions. Zeno R-50 provides more realistic expressions than other facially expressive robots such as KASPAR, but less realistic than FACE. The study aimed to compare expression recognition ability of ASD children with those of TD children. The study did not find any general deficiencies in expression recognition between groups, except for fear.

Although robots with nearly realistic expressive faces are considered as important achievements, they still suffer from several limitations. First, once the mechanical platforms are built, they are fixed and cannot be modified. Second, large numbers of actuators in the robots' face make them expensive and difficult to maintain. Finally, in the long term, some of the actuators either completely fail or weaken so the expressions are not as intense and recognizable.

A good solution for the problems mentioned above is rearprojected robots, which have received much attention recently $[25,26]$. Rear-projected robotic heads consist of a neck mechanism, a face-shaped translucent mask and a projector that projects a computer graphic avatar onto the mask. The computer graphic avatar is produced using character animation technologies. Compared to android robots, rearprojected robots are less expensive more flexible, and feature low power consumption and fast reaction time. Dome robot [27] is one of the rear-projected robots that uses a cartoonish animated face projected on a dome-shaped mask. Dome robot lacks a realistic human face. Another example of rearprojected robots is the Lighthead robotic face [28] which projects a more realistic animation onto a face-shaped translucent mask. Al Moubayed et al. presented Furhat [25], a human-like light-projected robot that uses computer animation to demonstrate facial expressions and a mirror to produce a side projection-angle which results in a larger form factor.

In this pilot study, we used Ryan Companionbot, a rearprojected humanoid robot developed by DreamFace technologies, to evaluate the facial expression recognition ability of ASD children compared to TD children. Our first hypothesis is that ASD children will perform worse than the TD control group on average. Our second hypothesis is that both groups will show a higher expression recognition accuracy as the intensity of Ryan's facial expressions increase. Our third hypothesis is that both groups will perform worse in recognizing negative expressions (i.e. anger, disgust, and fear) comparing to other expressions, as suggested by some studies [29]. Finally, we predict that Ryan's facial expressions will be, overall, comprehensible and recognizable with high average accuracy for children in both groups.

The rest of this paper is structured as follows. Section II describes the Ryan Companionbot specification, the research methodology, and a description of human subjects participated in the study. Section III presents the results and analyses. Section IV discusses the results and findings of this research. Finally, Section V concludes this paper. Future work is also discussed in this section.

\section{METHODS}

\section{A. Ryan Companionbot}

Ryan is a rear-projected humanoid robot developed at DreamFace Technologies, which is based on the Expressionbot [26]. It is created by using character animation technologies to show 3D avatar models that produce natural speech and facial expressions. The animated face model is then projected onto a face-shaped translucent mask. This design is not only an effective alternative to overcome many of the limitations with the mechanical-expressive face design, it also provides flexibility to redesign and customize facial expressions, from simplistic non-sophisticated expressions to nearly realistic human like expressions.

The 3D models of six universal basic expressions (i.e. anger, disgust, fear, happiness, sadness, and surprise) were designed in Maya based on the Facial Action Coding System (FACS) [30]. For example, sadness involves Inner Brow Raiser (AU 1), Brow Lowerer (AU 4) and Lip Corner Depressor (AU 15) and happiness involves Cheek Raiser (AU 6) and Lip Corner Puller (AU 12). Fig. 1 shows Ryan and six basic expressions demonstrated on its face with $100 \%$ intensity.

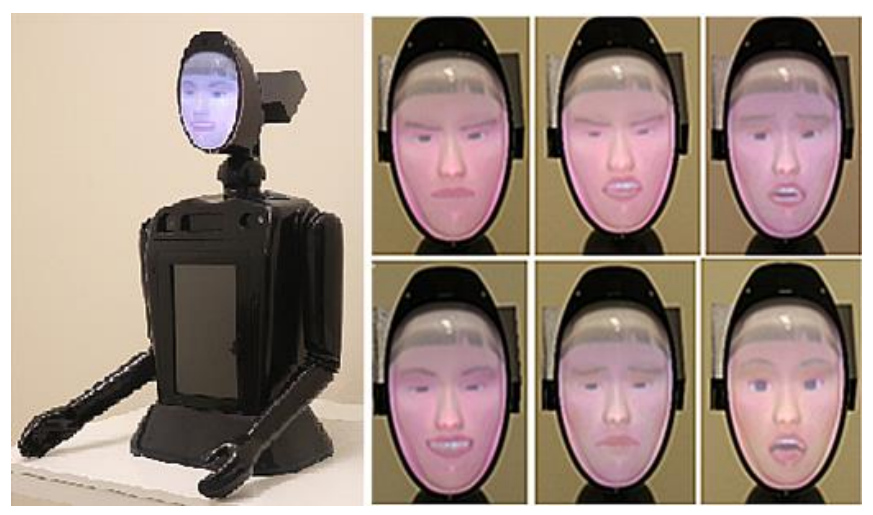

Figure 1. Left) Ryan Companionbot robot [31,32]. Right) Expressions demonstrated by Ryan with $100 \%$ intensity (Top from left to right: anger, disgust, fear. Bottom from left to right: happiness, sadness, surprise) 


\section{B. Experiment Design}

In this pilot study, Ryan demonstrated a sequence of facial expressions. The set consisted of six basic facial expressions (i.e. anger, disgust, fear, happiness, sadness, and surprise) and four different intensities (i.e. $25 \%, 50 \%, 75 \%$, and 100\%) for each of the expressions (total of 24 trials). Each expression intensity was determined based on the number of frames between neutral and $100 \%$ intensity of that specific expression [33]. (e.g. for $25 \%$ intensity, number of frames between neutral and $100 \%$ intensity were divided by 4). Each expression started from a neutral state and progressed to a desired expression at a certain intensity level. For each participant, the expression demonstration started with the lowest intensity (i.e. 25\%). In each intensity level, the expressions were shown randomly. The intensity increased to the next level after all the trials were completed for the current intensity level. After showing each expression, Ryan resumed demonstrating the final intensity and waited for the children's response. When the children were ready to answer, they verbally gave their answer to Ryan and the researcher recorded the response.

Before the experiment started, children were introduced to the whole experiment setup including the robot and different expressions. They could choose one of the seven choices available for each expression. Choices included six basic expressions and neutral. Although no neutral expression was included in the expression set, the children could choose neutral if the expression was ambiguous due to low intensity. The researcher made sure each of the choices was understandable for the children. Children had the choices printed on a paper in front of them during the session. At times when the children were indecisive about their guess, the final guess was taken as the official decision/answer.

\section{Experiment Setup}

The experiment was conducted in the social robotics laboratory at the University of Denver where an IRB approval was obtained, and all the children's parents signed a consent form. The study was presented to each child in a room with the presence of Ryan and a research assistant. Each participant was asked to sit on a chair in front of Ryan. Each time the researcher made sure that Ryan's face is in the same height as the children's face. Fig. 2 shows the room setup.

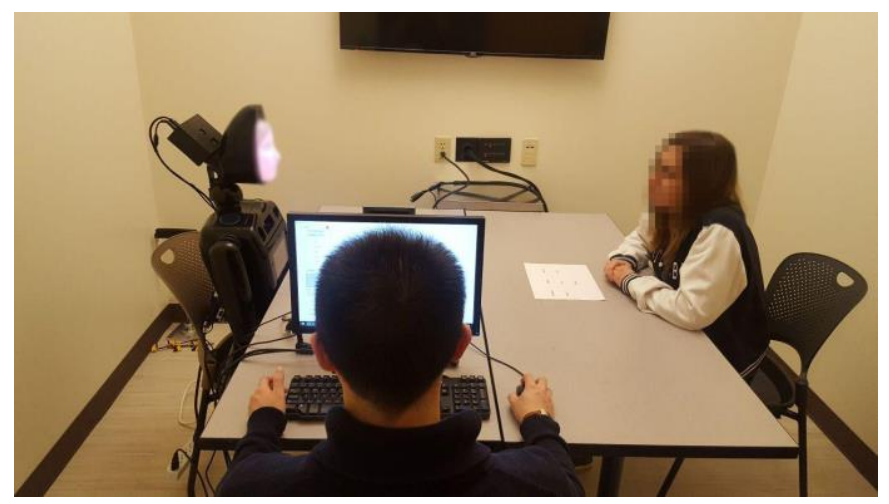

Figure 2. Room setup of the experiment protocol

\section{Participants}

Twelve children between the ages of 8 and 16 were recruited for the study. Six were classified as high functioning autistic by medical diagnosis (Age $M=11.1, S D=3.27$ ) (one female and five male) and six as typically developing children (Age $M=11.1, S D=3.12$ ) (six male).

In accepting high functioning ASD participants, it was insured that a doctor or psychiatrist formally diagnosed the children. Additionally, Autism Diagnosis Observation Schedule (ADOS) [34] examinations were performed by clinical psychologist collaborators in the Department of Psychology at the University of Denver to reassure that all the ASD participants met the threshold score for ASD diagnosis. As for the control group, neuro-typical children who had never been diagnosed with any kind of developmental or social disorder were recruited. Neuro-typical siblings of children with ASD were excluded from the study to ensure clear separation between the TD-control and ASD group.

Additionally, all the children's parents were asked to fill the Social Responsiveness Scale ${ }^{\mathrm{TM}}$ (SRS $\left.{ }^{\mathrm{TM}}\right)$ questionnaire, as a complementary assessment to the ADOS. According to the SRS diagnostic manual, a T-score between 60 and 75 indicates deficiencies in social skills that are associated with mild (high functioning) to moderate Autism Spectrum condition and a score above 76 indicates presence of deficiencies in social skills that are strongly associated with a clinical diagnosis of Autistic Disorder or Asperger's Disorder [35]. Of our six ASD participants, SRS scores were available for five of them. Comparing the scores for ASD participants $(M=66.4, S D=7.38)$ with TD control group $(M=40, S D=2.09)$ showed a significant difference $(t(5)=7.75, P<0.001)$ between the two groups.

\section{RESULTS}

Overall, we did not find a significant difference between the performances (average recognition accuracy) of the ASD $(M=0.71, S D=0.15)$ and TD $(M=0.73, S D=0.17)$ groups in expression recognition.

We ran a 3-way mixed ANOVA on recognition accuracy with group as a between-subject variable (ASD vs. TD) and expression (anger, disgust, fear, happiness, sadness and surprise) and intensity (25\%, 50\%, 75\% and 100\%) as withinsubject variables. The results revealed significant main effects of intensity $[F(3,240)=9.7, P<0.0001]$ and expression $[F(5,240)=6.5, P<0.0001]$ with no main effect of group. The three-way interactions between these factors was not significant, nor were the interactions between intensity and group, or between expression and the group.

The ANOVA analysis showed the main effects of expression and intensity but did not show any interaction between these factors by groups. We thus examine these factors in greater depth below, regardless of group.

Fig. 3 shows the recognition accuracy for each expression. The average recognition accuracy was lower for disgust and fear expressions. Our analysis shows that both groups performed significantly worse in recognizing disgust $(M=0.5$, 
$S D=0.3)$ versus the average of other expressions $(M=0.76$, $S D=0.15) \quad(t(16)=-2.7, \quad P=0.008)$. Also, the average performance of groups in recognizing fear $(M=0.54, S D=0.38)$ was significantly lower compared to the average of other expressions $(M=0.75, S D=0.14)(t(14)=-1.8, P=0.04)$.

Fig. 4 demonstrates the effect of increasing the intensity on recognition accuracy. Our analysis shows that increasing the intensity from $25 \%$ to $50 \%$ had a significant effect on recognition accuracy. The recognition accuracy with the $25 \%$ intensity $(M=0.5, S D=0.26)$ was significantly lower than the accuracy with $50 \%$ intensity $(M=0.72, S D=0.22)(t(11)=$ $3.75, P=0.001)$. Additionally, the recognition accuracy with the $75 \%$ intensity $(M=0.83, S D=0.14)$ was significantly higher than the accuracy with the $50 \%$ intensity $(t(11)=-2.34$, $P=0.019)$. We did not find any significant effect of the intensity increment on recognition accuracy from $75 \%$ to $100 \%$.

Fig. 5 and 6 show the confusion tables for the ASD participants and TD group, respectively. The figures compare the ability of both groups to recognize expressions and reveal how the demonstrated expressions by Ryan are recognizable by children. It can be seen that disgust and fear are more often mistaken with other expressions.

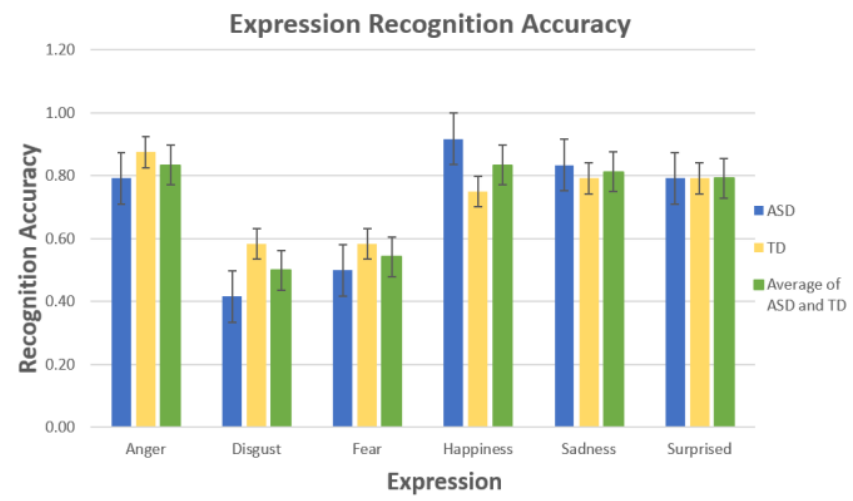

Figure 3. The average group accuracy is shown for both ASD (blue), TD (yellow), and the average of both groups (green). Each bar represents the average of the group for that specific expression. Error bars are standard errors.

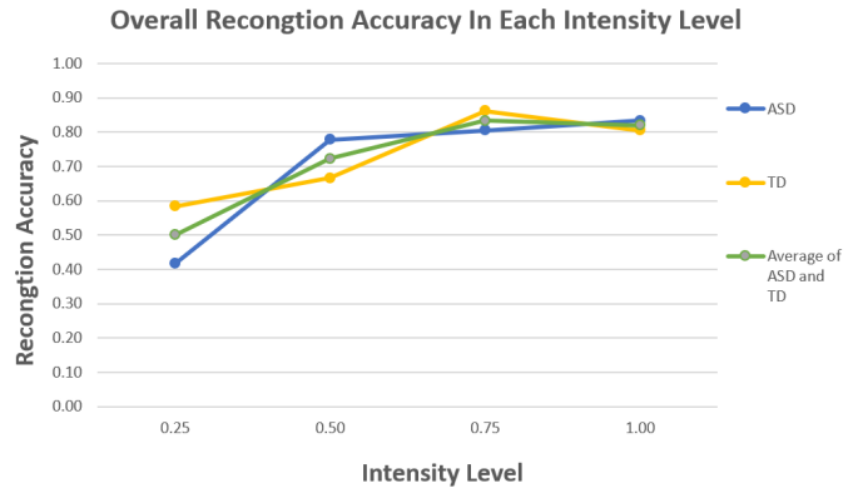

Figure 4. The average group accuracy is shown for both ASD (blue), TD (yellow), and the average of both groups in different intensity levels.

\begin{tabular}{|c|c|c|c|c|c|c|c|}
\hline ASD & Neutral & Anger & Digust & Fear & Happiness & Sad & Surprise \\
\hline Anger & 0.13 & $\mathbf{0 . 7 9}$ & 0 & 0 & 0 & 0.08 & 0 \\
\hline Digust & 0.04 & 0.33 & $\mathbf{0 . 4 2}$ & 0.08 & 0 & 0.08 & 0.04 \\
\hline Fear & 0.17 & 0 & 0.04 & $\mathbf{0 . 5}$ & 0 & 0.29 & 0 \\
\hline Happiness & 0.04 & 0 & 0 & 0 & $\mathbf{0 . 9 2}$ & 0 & 0.04 \\
\hline Sad & 0.17 & 0 & 0 & 0 & 0 & $\mathbf{0 . 8 3}$ & 0 \\
\hline Surprise & 0.08 & 0 & 0 & 0.04 & 0.04 & 0.04 & $\mathbf{0 . 7 9}$ \\
\hline
\end{tabular}

Figure 5. Confusion matrix for the recognition of six basic expressions by ASD group. Rows are ground truth and columns are recognized expressions by ASD participants.

\begin{tabular}{|c|c|c|c|c|c|c|c|}
\hline TD & Neutral & Anger & Digust & Fear & Happiness & Sad & Surprise \\
\hline Anger & 0.12 & $\mathbf{0 . 8 8}$ & 0 & 0 & 0 & 0 & 0 \\
\hline Digust & 0.08 & 0.21 & $\mathbf{0 . 5 8}$ & 0.04 & 0.04 & 0 & 0.04 \\
\hline Fear & 0.04 & 0 & 0.04 & $\mathbf{0 . 5 8}$ & 0.13 & 0.13 & 0.08 \\
\hline Happiness & 0.08 & 0 & 0.04 & 0 & $\mathbf{0 . 7 5}$ & 0 & 0.13 \\
\hline Sad & 0.17 & 0 & 0 & 0.04 & 0 & $\mathbf{0 . 7 9}$ & 0 \\
\hline Surprise & 0.17 & 0 & 0.04 & 0 & 0 & 0 & $\mathbf{0 . 7 9}$ \\
\hline
\end{tabular}

Figure 6. Confusion matrix for the recognition of six basic expressions by the TD control group. Rows indicate ground truth and columns indicate the proportion of expression categorizations made by TD participants.

\section{DISCUSSION}

In general, we did not find a general impairment in the ASD group for recognizing facial expressions of emotion. This could have occurred for several reasons. First, the sample size was small in each group. Second, since all the participants in this study were children with high functioning autism, they had higher levels of cognitive abilities. Thus, it is reasonable that they performed close to their TD peers. However, as mentioned before, the emotion recognition findings in ASD have been inconsistent and there are many studies $[15,16]$ that disagree with any general expression recognition deficiency in ASD children.

Although we did not find differences between groups, both groups showed significantly lower performance in recognizing disgust and fear expressions. This is consistent with some evidence that people with ASD may have particular deficits recognizing negative basic emotions [29]. For instance, studies have shown lower accuracy in recognizing fear [36,37] and disgust [38]. We found impairment in recognizing fear and disgust in both groups.

Moreover, there was a significant effect of increasing the intensity on the average recognition accuracy. The effect remained significant as the intensity increased up to $75 \%$. Since no interaction was found between expressions and intensity, it can be concluded that all the expressions demonstrated by Ryan are recognized with $80 \%$ accuracy and higher when the expression intensity level reaches $75 \%$ and higher.

Finally, Fig. 3 and Fig. 4 show that the recognition rates for most of the expressions are better in higher intensities, and in lower intensities such as $25 \%$ which is difficult to recognize the expression, Ryan was successful to effectively conveying the expressions. Fig. 5. shows that in the ASD group, disgust was often mistaken with anger. This low recognition accuracy might be due to inherent deficiency of ASD children in recognizing negative expressions as shown by other studies [37]; however, we did not find any difference between ASD 
and TD group in recognizing disgust. In general, the only expressions with low recognition accuracies are disgust and fear; besides, according to previous studies [36-38], children are expected to show lower recognition in these expressions. Therefore, Ryan can successfully demonstrate facial expressions and convey facial social cues to children. Anecdotally, all the children in both groups showed an acceptance toward Ryan when being first introduced to the robot, which confirms that Ryan is an effective tool to be used in future studies of SAR for children diagnosed with autism.

\section{CONCLUSION AND FUTURE WORK}

This paper presented a comparative pilot study on how children diagnosed with ASD compared to their TD peers can recognize expressions demonstrated by a rear-projected humanoid robot. We also studied the effect of using different intensities on the expression recognition accuracy. In a group of 12 participants, it was found that there was no significant impairment in the ASD group compared to the TD group in recognizing the basic facial expressions on average; so this study did not find any result to support our first hypothesis Moreover, as expected in the second hypothesis, a strong impairment for both groups was found in recognizing fear and disgust. Additional analysis of the results showed that increasing the intensity from $25 \%$ to $50 \%$ and to $75 \%$, significantly affects the expression recognition accuracy in both groups which supports the third hypothesis.

One take home lesson from this research is that a general assumption of impairment in expression recognition for children with ASD should not be assumed when designing SAR-based therapies for them. The findings of this study therefore support the results of other studies such as [23] that have shown individuals with ASD are overall successful in matching expressions in still images. Also, the capability of Ryan to successfully convey all the six basic facial expressions and its potential to be used in future studies of SAR was investigated. Furthermore, this study was not faced with any significant expression misrecognition due to defective or confusing expression demonstration by Ryan. Moreover, Ryan provides flexibility to redesign and customize facial expressions, from simplistic nonsophisticated expressions to nearly realistic human-like expressions, which make it a great choice for further SAR studies.

Finally, further work with more participants in each group and a greater number of trials should be done to address any deficiencies in expression recognition, if such exists. Last but not least, there needs to be further investigation on the ability of ASD children to match expressions with their meaning and mental states linked to them. One possible way is to study the affect the context in expression recognition. Through this work, we seek to contribute in the field of SAR and ASD research to develop more advanced therapies for social skills development.

\section{ACKNOWLEDGMENT}

This research is partially supported by grants IIS1450933 and CNS-1427872 from the National Science Foundation. We would like to thank Mr. Hojjat Abdollahi, a $\mathrm{PhD}$ student in the Computer Vision and Social Robotics Laboratory at the University of Denver, for his help to program the robot application.

\section{REFERENCES}

[1] Rogers, Sally J. "Developmental regression in autism spectrum disorders." Developmental Disabilities Research Reviews 10, no. 2 (2004): 139-143.

[2] Warren, Zachary, Melissa L. McPheeters, Nila Sathe, Jennifer H. Foss-Feig, Allison Glasser, and Jeremy Veenstra-VanderWeele. "A systematic review of early intensive intervention for autism spectrum disorders." Pediatrics 127, no. 5 (2011): e1303-e1311.

[3] American Psychiatric Association. Diagnostic and statistical manual of mental disorders (DSM-5®). American Psychiatric Pub, 2013.

[4] Uljarevic, Mirko, and Antonia Hamilton. "Recognition of emotions in autism: a formal meta-analysis." Journal of autism and developmental disorders 43, no. 7 (2013): 1517-1526.

[5] Ricks, Daniel J., and Mark B. Colton. "Trends and considerations in robot-assisted autism therapy." In Robotics and Automation (ICRA), 2010 IEEE International Conference on, pp. 4354-4359. IEEE, 2010.

[6] Feng, Huanghao, Hosein M. Golshan, and Mohammad H. Mahoor. "A Wavelet-based Approach to Emotion Classification using EDA Signals.” DOI > 10.1016/j.eswa.2018.06.014

[7] Kim, Elizabeth S., Rhea Paul, Frederick Shic, and Brian Scassellati. "Bridging the research gap: Making HRI useful to individuals with autism." Journal of Human-robot interaction 1, no. 1 (2012).

[8] Baron-Cohen, Simon. "The hyper-systemizing, assortative mating theory of autism." Progress in Neuro-Psychopharmacology and Biological Psychiatry 30, no. 5 (2006): 865-872.

[9] Wainer, Joshua, Ben Robins, Farshid Amirabdollahian, and Kerstin Dautenhahn. "Using the humanoid robot KASPAR to autonomously play triadic games and facilitate collaborative play among children with autism." IEEE Transactions on Autonomous Mental Development 6, no. 3 (2014): 183-199.

[10] Duquette, Audrey, François Michaud, and Henri Mercier. "Exploring the use of a mobile robot as an imitation agent with children with lowfunctioning autism." Autonomous Robots 24, no. 2 (2008): 147-157.

[11] Mazzei, Daniele, Lucia Billeci, Antonino Armato, Nicole Lazzeri, Antonio Cisternino, Giovanni Pioggia, Roberta Igliozzi, Filippo Muratori, Arti Ahluwalia, and Danilo De Rossi. "The face of autism." In RO-MAN, 2010 IEEE, pp. 791-796. IEEE, 2010.

[12] Hanson, David, Daniele Mazzei, Carolyn Garver, Arti Ahluwalia, Danilo De Rossi, Matt Stevenson, and Kellie Reynolds. "Realistic humanlike robots for treatment of ASD, social training, and research; shown to appeal to youths with ASD, cause physiological arousal, and increase human-to-human social engagement." In Proceedings of the 5th ACM International Conference on Pervasive Technologies Related to Assistive Environments (PETRA'12). 2012.

[13] Baron-Cohen, S., and Cecilia Heyes. "Mindblindness: An Essay on Autism and Theory of Mind." Nature 375, no. 6529 (1995): 290-290.

[14] Baron-Cohen, Simon, et al. "The amygdala theory of autism." Neuroscience \& Biobehavioral Reviews 24.3 (2000): 355-364.

[15] Ozonoff, Sally, Bruce F. Pennington, and Sally J. Rogers. "Are there emotion perception deficits in young autistic children?" Journal of Child Psychology and Psychiatry 31, no. 3 (1990): 343-361.

[16] Castelli, Fulvia. "Understanding emotions from standardized facial expressions in autism and normal development." Autism 9, no. 4 (2005): 428-449.

[17] Ekman, Paul. "Are there basic emotions?" (1992): 550.

[18] Smith, Miriam J. Law, Barbara Montagne, David I. Perrett, Michael Gill, and Louise Gallagher. "Detecting subtle facial emotion 
recognition deficits in high-functioning autism using dynamic stimuli of varying intensities." Neuropsychologia 48, no. 9 (2010): 2777-2781.

[19] Kozima, Hideki, Marek P. Michalowski, and Cocoro Nakagawa. "Keepon." International Journal of Social Robotics 1.1 (2009): 3-18.

[20] Kozima, Hideki, Cocoro Nakagawa, and Yuriko Yasuda. "Childrenrobot interaction: a pilot study in autism therapy." Progress in Brain Research 164 (2007): 385-400.

[21] Robins, Ben, Kerstin Dautenhahn, and Paul Dickerson. "From isolation to communication: a case study evaluation of robot assisted play for children with autism with a minimally expressive humanoid robot." In Advances in Computer-Human Interactions, 2009. ACHI'09. Second International Conferences on, pp. 205-211. IEEE, 2009.

[22] Pioggia, Giovanni, M. L. Sica, Marcello Ferro, Roberta Igliozzi, Filippo Muratori, Arti Ahluwalia, and Danilo De Rossi. "Human-robot interaction in autism: FACE, an android-based social therapy." In Robot and Human interactive Communication, 2007. RO-MAN 2007. The 16th IEEE International Symposium on, pp. 605-612. IEEE, 2007.

[23] Salvador, Michelle J., Sophia Silver, and Mohammad H. Mahoor. "An emotion recognition comparative study of autistic and typicallydeveloping children using the zeno robot." In Robotics and Automation (ICRA), 2015 IEEE International Conference on, pp. 6128-6133. IEEE, 2015.

[24] Salvador, Michelle, Anna Sophia Marsh, Anibal Gutierrez, and Mohammad H. Mahoor. "Development of an ABA Autism Intervention Delivered by a Humanoid Robot." In International Conference on Social Robotics, pp. 551-560. Springer, Cham, 2016.

[25] MOUBAYED, SAMER AL, Gabriel Skantze, and Jonas Beskow. "The furhat back-projected humanoid head-lip reading, gaze and multi-party interaction." International Journal of Humanoid Robotics 10, no. 01 (2013): 1350005.

[26] Mollahosseini, Ali, Gabriel Graitzer, Eric Borts, Stephen Conyers, Richard M. Voyles, Ronald Cole, and Mohammad H. Mahoor. "Expressionbot: An emotive lifelike robotic face for face-to-face communication." In Humanoid Robots (Humanoids), 2014 14th IEEERAS International Conference on, pp. 1098-1103. IEEE, 2014.

[27] Hashimoto, Minoru, and Daisuke Morooka. "Facial expression of a robot using a curved surface display." In Intelligent Robots and Systems, 2005.(IROS 2005). 2005 IEEE/RSJ International Conference on, pp. 765-770. IEEE, 2005.

[28] Delaunay, Frédéric, Joachim De Greeff, and Tony Belpaeme. "Towards retro-projected robot faces: an alternative to mechatronic and android faces." In Robot and Human Interactive Communication, 2009. ROMAN 2009. The 18th IEEE International Symposium on, pp. 306-311. IEEE, 2009.

[29] Ashwin, Chris, Emma Chapman, Livia Colle, and Simon Baron-Cohen. "Impaired recognition of negative basic emotions in autism: A test of the amygdala theory." Social neuroscience 1, no. 3-4 (2006): 349-363.

[30] Ekman, Paul, and Erika L. Rosenberg, eds. What the face reveals: Basic and applied studies of spontaneous expression using the Facial Action Coding System (FACS). Oxford University Press, USA, 1997.

[31] Abdollahi, Hojjat, Ali Mollahosseini, Josh T. Lane, and Mohammad H. Mahoor. "A Pilot Study on Using an Intelligent Life-like Robot as a Companion for Elderly Individuals with Dementia and Depression." arXiv preprint arXiv:1712.02881 (2017).

[32] Mollahosseini, Ali, Hojjat Abdollahi, and Mohammad $\mathrm{H}$. Mahoor."Studying Effects of Incorporating Automated Affect Perception with Spoken Dialog in Social Robots" Robot and Human Interactive Communication (RO-MAN), 2018 27th IEEE International Symposium on. IEEE, 2018.

[33] Mollahosseini, Ali, Hojjat Abdollahi, Timothy D. Sweeny, Ron Cole, and Mohammad H. Mahoor. "Role of embodiment and presence in human perception of robots' facial cues." International Journal of Human-Computer Studies 116 (2018): 25-39.

[34] Lord, Catherine, Michael Rutter, Pamela C. DiLavore, and Susan Risi. "Autism diagnostic observation schedule-WPS (ADOS-WPS)." Los Angeles, CA: Western Psychological Services (1999).

[35] "Social Responsiveness Scale (SRS) - WPS", Wpspublish.com, 2016. [Online]. Available: http://www.wpspublish.com/store/p/2993/social-responsivenessscalesrs-by-john-n-constantino-md. [Accessed: 08- Mar- 2016].

[36] Howard, M. A., P. E. Cowell, J. Boucher, P. Broks, A. Mayes, A. Farrant, and N. Roberts. "Convergent neuroanatomical and behavioural evidence of an amygdala hypothesis of autism." Neuroreport 11, no. 13 (2000): 2931-2935.

[37] Pelphrey, Kevin A., Noah J. Sasson, J. Steven Reznick, Gregory Paul, Barbara D. Goldman, and Joseph Piven. "Visual scanning of faces in autism." Journal of autism and developmental disorders 32, no. 4 (2002): 249-261.

[38] Golan, Ofer, Yana Sinai-Gavrilov, and Simon Baron-Cohen. "The Cambridge Mindreading Face-Voice Battery for Children (CAM-C): complex emotion recognition in children with and without autism spectrum conditions." Molecular autism 6, no. 1 (2015): 22. 